\title{
Therapeutic Applications of Engineered Chimeric Antigen Receptors-T cell for Cancer Therapy
}

\author{
Amina Hussain \\ Department of Environmental Sciences, Fatima, Jinnah, Woman University \\ Rawalpindi
}

\begin{abstract}
Findings of new targeted treatments with adequate safety evaluations is essential for better cancer cures and mortality rates. Immunotherapy holds promise for patients with relapsed disease, with the ability to elicit long-term remissions. Emerging promising clinical results in B-cell malignancy using gene-altered T-lymphocytes uttering chimeric antigen receptors have sparked a lot of interest. This treatment could open the path for a major difference in the way we treat tumors that are resistant or recurring. Genetically altered $\mathrm{T}$ cells used to produce tumor-specific chimeric antigen receptors are resurrected field of adoptive cell therapy by demonstrating remarkable success in the treatment of malignant tumors. Because of the molecular complexity of chimeric antigen receptors - T cells, a variety of engineering approaches to improve safety and effectiveness are necessary to realize larger therapeutic uses. In this study, we investigate at new strategies for enhancing chimeric antigen receptors-T cell therapy by altering chimeric antigen receptors proteins, T lymphocytes, and their relations with other solid tumor microenvironment (TME) aspects.
\end{abstract}

Key Words : chimeric antigen receptors-T cell; solid tumor microenvironment; cancer therapy

\section{INTRODUCTION}

T-cells can recognise tumor-associated antigens without using the major histocompatibility complex if they have a chimeric antigen receptor made artificially [1]. In the therapy of resistant B cell malignancies, chimeric antigen receptor-T cells that targeted the pan-B-cell marker CD19 showed an excellent reaction [2]. To overcome tumor defensive pathways such as antigen resistance, physiological hurdles and immunosuppressive entrance into solid tumors, creating 
appropriate chimeric antigen receptor - T immunotherapy for non-B-cell cancers has necessitated more technically complicated techniques [3]. We look at current and future chimeric antigen receptors design methods, tumor-specific $\mathrm{T}$ lymphocytes receptors, and how designed $\mathrm{T}$ lymphocytes interface with the tumour microenvironment shown in Figure 1. The purpose of this research is to increase the efficacy and security of adoptive T cell treatment for solid tumours.

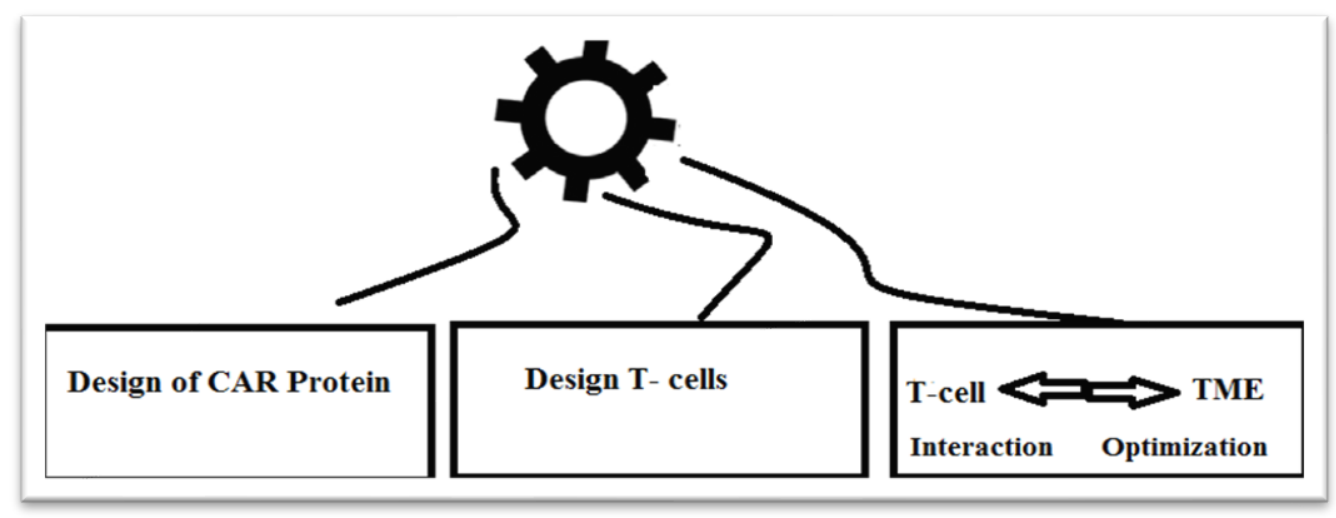

Fig 1. Approaches for enhancing the activity of chimeric antigen receptor $-\mathrm{T}$ cells in solid tumours

\section{Development in Chimeric Antigen Receptor Designs}

The first brilliant idea for combining antibody-type specificity with T-lymphocyte activation was to combine the constant area of a T-cell receptor to the variable regions of a bacterial antigenrecognizing antibody [4]. Single-chain variable fragments are still widely seen as the exogenous antigen-sensing domain in chimeric antigen receptors. They're made up of a flexible binder that joins a monoclonal antibody's variable heavy and light chains[5].

Antigens identified by Single-chain variable fragments linked to the CD3z activation domain may produce tumor-specific toxicity, according to the first tests using tumor-targeting chimeric antigen receptors. In the great majority of instances, T cells produce these " 1 st generation" chimeric antigen receptors, which only would include the CD3z chain for T-lymphocytes signaling, unsuccessful to provoke substantial antitumor reactions [6]. 2nd - and 3rd generation chimeric antigen receptors with one or two costimula were produced after it was recognized that the endogenous T-cell receptor required connotation with the other costimulatory or adjunct molecules for vigorous signaling [7]. 
These costimulatory domains, which are primarily derivative from CD28, improved overall cytotoxicity, cytokine secretion, and chimeric antigen receptors-T cell tumor development and persistence [8]. The costimulatory domain employed has an effect on a lot of things, namely metabolic activities, T-cell memory development, and antigen-independent regulatory stimulation, all of which are essential driving variables[9].

The release of the anti-inflammatory cytokine interleukin (IL)-10 was inhibited by a thirdgeneration chimeric antigen receptors containing OX40 and CD28 costimulatory domains. Chimeric antigen receptors of the fourth generation, named "armored" chimeric antigen receptors because of their extra stimulation domains, have recently been developed. Chimeric antigen receptors - $\mathrm{T}$ cells that generate pro-inflammatory cytokines are known as $\mathrm{T}$ cells redirected for ubiquitous cytokine-mediated killing (TRUCK) [10].

The ability of soluble agents like IL18 or IL-15 to increase T - cell activation, as well as a combination of CCL19 and IL-7 to recruit endogenous immune system and trigger cancer memory reactions, has been studied [11]. Advances in chimeric antigen receptors design have permitted much more optimization of each of the four primary components of a chimeric antigen receptors hinged domains or extracellular spacer, extracellular antigen-sensing domains, intracellular signaling domains and the single trans membranes, enabling chimeric antigen receptors therapy to progress [12].

\section{Logic-Gated T cell Activation through Combinatorial Antigen Sensing}

Chimeric antigen receptors-T cells have improved their stability and anticancer efficiency by identifying many antigens in combination. To trigger a chimeric antigen receptors-T cell, ANDgate logic necessitates the coexistence of several antigens, decreasing the threat of off-target recognition or toxicities that kill healthy cells [13]. The synthetic Notch (synNotch) receptor was intended to measure a TAA and trigger production of a chimeric antigen receptors, which can then induce T-lymphocytes response when secondary TAA is detected[14].

When the non-tumor target is not close to the tumor cells, this method has been demonstrated to reduce inflammatory reactions [15]. Because synNotch recognizes TAA \#1 before chimeric antigen receptors recognizes TAA \#2, TAA \#1 from a malignant cells could trigger a T cell's synNotch receptor, which then targets a normal cell producing TAA \#2. In alternate AND-gate 
technique, $\mathrm{CD} 3 \mathrm{z}$ chain and costimulatory domain split into binary distinct receptors, to each one recognizes the other [16].

This technique, though, is susceptible to "leakiness" because 1st generation chimeric antigen receptors that just incorporate the $\mathrm{CD} 3 \mathrm{z}$ chain already are proficient of signaling. Another way instructs $\mathrm{T}$ cells release a specifically active cytotoxic proteins in response to TAA \#1 on the surface of the cell getting identified by chimeric antigen receptors or TCR; the designed protein gets cytotoxic only if TAA \#2 within the cell is identified[17]. OR-gate logic was used to improve therapeutic impact by preventing antigen spillage and carcinoma cells loss of the target epitope [18] .

Chimeric antigen receptors -T cells designed to use AND-NOT logic can also assist protect normal tissue from toxicity. This method combines an inhibiting chimeric antigen receptors that target a TAA with an activation chimeric antigen receptors that targeting an antigen present on normal tissue[19]. A prostate-specific membranes antigen targeting inhibiting chimeric antigen receptors with the leading to apoptosis protein. Although AND and AND-NOT logic can enhance chimeric antigen receptors - $\mathrm{T}$ cell protection by enhancing specificity, OR-gate logic used to increase antitumor effect in order to avoid leakage of antigen, or tumors cells losing targeting epitope [20]. An OR-gate chimeric antigen receptors can identify two distinct TAAs, and attaching to one of them activates T cells. One OR-gate technique makes use of a pooled combination of two chimeric antigen receptors - T cell populations (chimeric antigen receptors pool), each of which expresses a monospecific chimeric antigen receptors. A variation on this topic is to give two separate chimeric antigen receptors $-\mathrm{T}$ cell products at the same time[21]. 1 (PD1inhibitorysignalingdomain was coexpressed with a second-generation CD19 inhibiting chimeric antigen receptors in a solid evidence research, and the inhibiting chimeric antigen receptors suppressed chimeric antigen receptors-T cell stimulation in the vicinity of PSMA [22]. Coexpression of two distinct chimeric antigen receptors in each T cell (dual chimeric antigen receptors) is another technique[23]. Another option employs tandem bispecific chimeric antigen receptors (Tan- chimeric antigen receptors), which consist of splited single receptor chain on $2 \mathrm{scFv}$ domains, and has been proven to be substantially better both to the chimeric antigen receptors pool and dual- chimeric antigen receptors strategies[24]. CD19-CD20 and CD19-CD22 bispecific chimeric antigen receptors, in 
example, have been studied in the treatment of B-cell malignancies and are currently being evaluated in clinical trials for myeloma and lymphocytic leukemi respectively [25].

\section{Controllability and Safety using ON/OFF Switches}

Self-regulating on/off switches have also been utilised to increase the security and adaptability of chimeric antigen receptor modifications. Chimeric antigen receptors - T cells that have previously been employed are "always on." When it comes to chimeric antigen receptors derived cytotoxicity, however, this isn't necessarily the greatest solution. Patients treated with chimeric antigen receptors-T cells have reported a variety of systemic adverse effects in adding to off-target damage. A common occurrence is cytokine release syndrome (CRS). However, when it comes to chimeric antigen receptors derived cytotoxicity, this isn't always the best option[15]. Patients who got chimeric antigen receptors - $\mathrm{T}$ cells had a variety of systemically adverse effects in additional to the target-off and "target on, tumor-off" toxicity mentioned above[26]. Cytokine release syndrome is a common occurrence (CRS). Changing the structure of the chimeric antigen receptors protein can access the available of functional chimeric antigen receptors, allowing the receptor to be signaling-competent only under specified conditions, rather than modifying protein half-life. For example, chimeric antigen receptors have an antigen-binding region that can be masked by a tailored inhibiting peptide, allowing them to function only when the inhibiting peptide is removed [27].

\section{Chimeric antigen receptors that are Adapter-Dependent}

$\mathrm{T}$ cells can also be genetically manipulated to generate a receptor that requires the addition of a second protein component before it can translate antigen detection into T-cell activation [28]. A biotin-binding region is coupled to an intracellular T-cell triggering region in a "ubiquitous" receptor. T cells that express this biotin-specific receptors may theoretically be employed to attack a target cell that has been biotinylated using an antibody. Two of the technology's primary advantages are the capacity to manage the ON/OFF condition of $\mathrm{T}$ lymphocytes by giving or deferring biotinylated antibody, as well as the capacity to use biotin-specific receptor to attack a wide variety of TAAs by altering the responsiveness of the biotinylated antibody [29]. The idea of using a broad chimeric antigen receptor in combination with one or more antigen-targeting adaptor proteins to combat tumour variations and minimise adverse effects quickly caught on, resulting in a slew of new adapter-dependent chimeric antigen receptor designs, as well as a tiny adapter that 
binds to both FITC and folate and alleviates CRS-like harmful effects in NSG-treated rat models [30]. The SUPRA chimeric antigen receptors system is based on the idea of overexpressing a ubiquitous receptor on T-cells surfaces in combination with an extrinsically administered adaptor protein, which can develop various boolean logic gates in designed T-cells by displaying various receptors, each contain various adaptor protein associates recreated by the leucine-zipper dime [31].

Using AND-NOT or AND gates, researchers can increase sensitivity and cure tumour diversity by treating numerous antigens with different adaptor proteins [29]. Multi-component processes, on the other hand, have a larger range of factors to optimize, such as the adapter protein's half-life, bioavailability, and interface kinetics, as well as the base receptors, adaptor protein's, altered $\mathrm{T}$ cell's half-life, bio - availability, and interfaces kinetics[32]. As a result, it is unclear whether adaptor-dependent chimeric antigen receptors designs' sophisticated signal processing will convert into effective medicinal possibilities[33].

\section{Combinatorial Antigen Recognition}

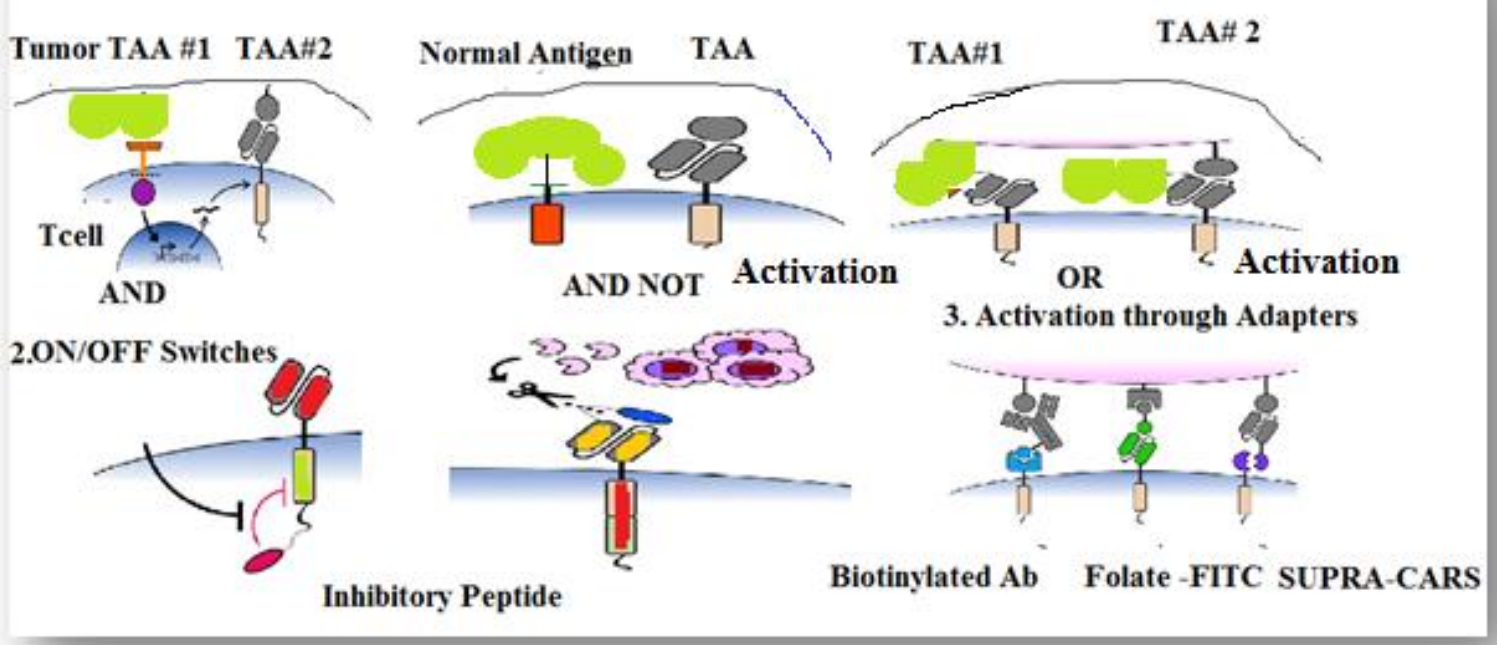

Fig 2. Protein designing methodologies to enhance the safety, program and effectiveness of chimeric antigen receptor 1. Antigen recognition via AND and AND-NOT logic, respectively, can improve binding ability and efficiency.2. Chimeric antigen receptor - $T$ cell activity may be rapidly and effectively changed with ON/OFF switches 3. Chimeric antigen receptors to activate only when an adapter is assembled can provide chimeric antigen receptor - $\mathrm{T}$ cells more control over their activity. 


\section{DESIGNING CAR EXPRESSING CELL}

\section{Designing Allogeneic Compatibility and Site-Specific chimeric antigen receptors Transgene Insertion}

Synthesized inducible promoters are utilized in the aforementioned cases of regulatory expression of genes, T-cell genomes frequently contain a gene-expression cassette, employing lentiviral and retroviral vectors, resulting in a range of integration sites and copy counts[34]. Integrating the transgenic into specific genomic regions is another way for establishing dynamic chimeric antigen receptors levels of expression [34]. CRISPR and CRISPR-associated protein 9 are two geneediting techniques (Cas9). T-cell designing has become possible mainly to the use of zinc finger nucleases (ZNFs) and activator-like effector nucleases (TALENs)[23]. Chimeric antigen receptor -T cells wipe out retrovirus-mediated random chimeric antigen receptor -transgene integration after CRISPR-Cas9-mediated incorporation of the CD19 chimeric antigen receptor transgenic into the TRAC loci [35]. The CD19 chimeric antigen receptor transgene was introduced into TRAC region using CRISPR-Cas9, resulting in chimeric antigen receptor T cells with enhanced in vivo studies[36]. However, new information suggests that whether site-specific chimeric antigen receptor insertion into TRAC loci improves T-cell activation is dependent on the chimeric antigen receptor design employed. Allogeneic T-cell therapy has also been made easier due to gene-editing techniques[37].

T-cell products obtained from better and healthier people can help resolve some of the challenges that arise with producing autologous cell therapy, such as getting enough high-quality $\mathrm{T}$ cells from individuals who have been heavily pretreated and have chronic illness[38]. Allogeneic T-cell transplant, on either hand, is a fairly new concept. Allogeneic T-cell transfer has been proposed to limit allograft resistance by including the deletion class-I major histocompatibility complex and endogenous TCR to avoid graft-versus-host disease [38]. ZFN-mediated suppression of HLA-A or TCRab expression in CD19 chimeric antigen receptor -T cells was used to achieve this[39]. Gene editing has been utilised to defend against GvHD in addition to avoiding the disease[40]. Before injecting chimeric antigen receptor-T cells, lymphodepletion is a typical preconditioning method boost the efficiency of the transplanted cells endogenous gene deletion and chimeric antigen receptors transgenic injection were conducted separately in the work mentioned above, resulting in a wide range of chimeric antigen receptor - $\mathrm{T}$ cells[41]. 
By inserting a single-guide RNA into chimeric antigen receptors U3 domain encoding lentiviral vector's 30 long terminal repeat sequence, the scientists were able to combine genome - editing and chimeric antigen receptors integration. Following Cas9 mRNA was electroporated, magnetic resonance imaging was employed [42].

\section{Negative regulators are eliminated}

Negative T-cell regulators' expression can also be suppressed via gene-editing approaches. Immune-checkpoint ligands are generally upregulated in tumour cells[43]. T-cell action is evaluated in the tumor microenvironment when cytotoxic T-cells -associated antigen (CTLAA) receptors PD-1 AND CTLA-4 are activated on T lymphocytes, inhibits the activity. In recent times, antibodies have been utilized to impair immunological checkpoints [44]. On modified T cells, checkpoint suppression can also be substituted by deletion of checkpoint receptor expression[45]. Several knockouts employing CRISPR-Cas9 in chimeric antigen receptor - T cells have been shown in numerous studies to reduce alloreactivity while also improving T-cell functionality [46]. Cas9:sgRNA ribonucleoprotein (RNP) complexed with mRNA expressing was electroporated into chimeric antigen receptor - $\mathrm{T}$ cells cells[14].

\section{Switching from an inhibited to a stimulatory mode of receptors}

Despite having shown tremendous therapeutic effectiveness against hematologic malignancies, chimeric antigen receptor -T cells have been limited in their ability to treat specific solid tumours due to a variety of obstacles. Furthermore, tumor cells induce tumorigenic and antigen heterogeneity TME immunosuppressive factors, and this inhibiting environment is exacerbated by immunosuppressive cells MDSCs and Tregs are examples of these forms of cells [47]. Among the transforming growth factor-b is a predominantly immunosuppressive soluble factor identified in the TME. T-cell differentiation into Tregs is triggered by this powerful cytokine as well as the immunosuppressive polarisation of macrophages phenotype M2[48]. The development of a chimeric antigen receptor that responds to transforming growth factor-b showed that chimeric antigen receptors usage to identify a soluble feature and T-cells are rearranged to transform inhibitory signals into antitumor activity signals[49]. Transforming growth factor-b responsive chimeric antigen receptor -T cells multiply in the presence of soluble transforming growth factor$\mathrm{b}$ and generate cytokines related with thelper cells type 1 (Th1). A combination of transforming 
growth factor-b internalization and paracrine Th1 cell stimulation protects neighbouring cells against transforming growth factor-inhibitory effects. [50]. '

Switch receptors, which are chimaeras made up of an extracellular domain that attaches to a repressive protein and an endodomain that controls the modulatory path, can be utilised to rewire signals, for example IL-4 is a kind of cytokine performing numerous functions inside body. [51]. Tumor microenvironment functions include promoting tumour development and producing M2 polarisation, suppressing the development of malignant cells effector T lymphocytes[52]. IL-4Ra in IL-4 receptors ectodomain is linked to either the IL-4 receptor or the IL-4Rb receptor ectodomain in IL-4 switching receptor [53].The bc receptor subunits or IL-7Ra endodomain similar to IL2 and IL-15 transmission has been reported to increase T-cell multiplication in the existence of IL-4 [54]. As a consequence, chimeric antigen receptor -T cells with coexpression of the IL4Ra:bC switching receptor had better cytotoxic capability [55].

\section{Chimeric antigen receptor -T Cells and Cancer Cells in Tumor Microenvironment}

Chimeric antigen receptor - T cells' limited therapeutic efficiency in solid tumours is due to the TME's immunosuppression properties[56]. Solid tumor microenvironment is characterized by physical barriers to immune cell invasion by tumours, elevated checkpoint receptors, and a protumor stromal microenvironment, all of which have been thoroughly discussed elsewhere, an excess of immunosuppression and pro-metastatic soluble molecules, a pro-tumor stromal microenvironment, an overabundance of immunosuppression, pro-metastatic soluble chemicals, and increased production of chemokines that primarily recruit immunosuppression leukocytes[57]. Researchers are striving to improve the development of tumor microenvironment responsive chimeric antigen receptor-T cells resulting of these characteristics [57].

\section{Penetration and Tumor Homing}

The effectiveness of chimeric antigen receptor - T cell treatment in solid tumours is dramatically reduced due to decreased immune activation [58]. Chemokine axis regulate $\mathrm{T}$ cell migration. Tumor cells may influence tumor-associated cell chemokine expression and up - regulation or down regulation inflammatory cytokines, leads to poor chimeric antigen receptor - $\mathrm{T}$ cell attraction[59]. Chimeric antigen receptor - T cells that are designed to regulate the chemokine 
receptors expression that are highly activated in the tumor microenvironment can turn a cancer defense pathway against it [60].

CCR2b, the main subtype of the chemokine receptor CCL2, has been transformed into chimeric antigen receptor -T cells that target GD2 and mesothelin, subsequent in improved T lymphocytes adhesion to CCL2-expressing malignant glioblastoma and mesothemia [61]. The better structured extracellular matrix associated to solid-tumors nodules prevents chimeric antigen receptor -T cells from accessing the cancer site. As a result, transgenic chimeric antigen receptor- $\mathrm{T}$ cells that produce heparinase, an anticoagulant, have been developed [62].

Chimeric antigen receptor - $\mathrm{T}$ cell activation can be aided or hindered by cytokines, which are signaling substances. Co-expression of chimeric antigen receptors with immunomodulatory cytokines may have a big impact. It is possible to improve chimeric antigen receptor -T cell growth, survivability, and mediator activity [63]. The immune system is inhibited by the microenvironment of solid tumors. For example, T-cells coexpress a CD19-targeting chimeric antigen receptor-T cells that generate CD19-targeting chimeric antigen receptors plus IL-2, IL-7, IL-15, or IL-21 inhibit tumors more efficiently in vivo than $\mathrm{T}$ lymphocytes the chimeric antigen receptors individually. Surprisingly, the gamma subunit is found in all four cytokine receptor complexes. On the proliferative chain, each cytokine had a distinct effect (gc) [64]. T-cells that generate IL12, IL-15, IL-18, and IL-21 in association with chimeric antigen receptors that targets a number of antigens have also been reported to be more active, multiply, and/or persist in vivo. But in the other hand, persistent overexpression of immunomodulatory cytokines can worsen damage [65].

\section{Disruption of immune-suppressive axes}

TME is rich in immune-checkpoint receptor and mediators including PD-L1 and PD-1, which actually reduce chimeric antigen receptor -T cell cytotoxic effects and cause anergy [66]. Immune checkpoint suppression has been shown in various studies to have a substantial synergistic impact with chimeric antigen receptor - T cell treatment (Huemer et al., 2017). A combined therapy combining chimeric antigen receptor $-\mathrm{T}$ cells and externally given checkpoint inhibitors is now being investigated for the treatment. Chimeric antigen receptor - $\mathrm{T}$ cells are sometimes used to develop immune-checkpoint blockers such anti-PD-1 scFvs and anti-PD-L1 antibody, and the ability to express PD-1 receptors that are notably negative (DNRs) [68]. 
Tumor development and chimeric antigen receptor - $\mathrm{T}$ cell therapy resistance are connected to aberrant cytokine expression in the TME. Transforming growth factor -b interacts with cancer cells, stroma, and both to contribute a complex role in cancer development [66]. Adaptive and innate immune cells secrete chemokines, cytokines, and growth factors that weaken the immune system; (2) ECM remodeling (3) macrophage, neutrophil, and monocyte immunosuppressive T cells; and (4) prevented $\mathrm{T}$ cells multiplying. Chimeric antigen receptor $-\mathrm{T}$ cells have been developed to produce a transforming growth factor $-\mathrm{b}$ receptor to counteract these exceptional effects [14]. DNR suppresses endogenous transforming growth factor -b signalling in a prostate cancer model, resulting in enhanced $\mathrm{T}$ cell proliferation and antitumor effectiveness. These findings prompted the activation of T cells in a new therapy trial for the prevention of chronic and resistant metastatic prostate cancer that coexpressed PSMA chimeric antigen receptors and the DNR [69]. Unlike transforming growth factor -b-targeting chimeric antigen receptors and transforming growth factor -b switch receptors, the DNR does not send a signal to the transformed $\mathrm{T}$ cell. It has been shown that the anticancer potential of CD19-targeting chimeric antigen receptor -T cells that additionally expressed mbaIL6, a non-signaling, membrane-bound IL-6 receptor, is maintained in vivo. However, it's unclear whether these chimeric antigen receptor - $\mathrm{T}$ cells can prevent CRS [70].

\section{Promoting Endogenous Immuno Responses by Restructuring TMEs}

To promote immune regulation or repression, tumours have acquired the ability of recruiting or rejecting particular types of leukocytes, such as chimeric antigen receptor -T cells. Additionally, cancer is on the rise it can cause an pro-metastatic phenotypic or immunosuppression in localized stroma, in addition to anti-inflammatory or phenotypic defects in localised lymphocytes [71].

Redesigning the tumor-cellular composition and reversing the immunosuppressive cell niche phenotype are two further ways to enhance the chimeric antigen receptor - $\mathrm{T}$ cell treatment efficiency[72]. To do this, designed chimeric antigen receptor -T cells to release cytokines or other soluble chemicals that promote paracrine or endocrine TME modification[73].

In syngeneic animal models, designed chimeric antigen receptor-T cells that generate IL-12 were able to redesign the tumour microenvironment by reprogramming TAMs that corresponds to M1 phenotype and lowering frequency of Tregs and MDSCs [57]. Chimeric antigen receptor-T cells that release IL-18 on a regular basis can change the organisation of the tumour microenvironment 
by boosting M1 macrophages, activated DC, and provoked NK cells[74]. The number of M2 macrophages and Treg cells is decreasing, whereas the number of cells is increasing [65].

In a study, IL-12-producing chimeric antigen receptor-T cells were compared to IL-18-producing chimeric antigen receptor-T cells. In a syngeneic murine model, IL-18 is more efficient at changing the immunosuppression tumour microenvironment [75]. Tumor microenvironment can be regulated using chimeric antigen receptor - $\mathrm{T}$ cells, which also create proinflammatory ligands that bind to the cell's surface [76]. CD40L, for example, is frequently expressed transiently on T cells. After activation of the TCR and contact with the CD40 receptor, a variety of types of immune cells can trigger APCs[77].

BiTEs, or bispecific T-cell engagers, are formed by two scFvs linked together, can be generated by chimeric antigen receptor - $\mathrm{T}$ cells to induce non-engineered T-cells to encounter cancer cells, as well as CD40+ cancer cell apoptosis and DC licencing [78]. The researchers created bispecific T-cell engagers, one with CD3 activation on T-cells and other scFv EGFR targeting, which is found in glioma cells, according to the researchers. [78]. In a number of ways, chimeric antigen receptor -T cells targeted EGFRvIII that create EGFR/CD3 bispecific T-cell engagers have been shown to eradicate orthotopic cancer xenograft models [79].

\section{Conclusion}

Hematologic malignancies have showed considerable potential with chimeric antigen receptor -T cell treatment. Solid tumors, on the other hand, present distinct obstacles that necessitate more advanced technologies to treat these refractory cancers magnificently. Recent developments in protein and cell editing have produced some remarkable discoveries. T cells' intrinsic fitness has been improved, and their anticancer function is on the rise. Despite the fact that the majority of engineering solutions published to date have centered on providing individual results. Advances in genome-editing technologies and genomic circuitry development have paved the way for multilayered strategies to T-cell therapeutic strategies that can simultaneously address multiple critical demands. Cancer cells and other malignant cells components, as well as synthetic and endogenous immune cells, must all be examined carefully. When it comes to chimeric antigen receptor - T cell clinical translation, to be employed in the treatment of solid tumors, it's critical to achieve a balance. These achievements vastly improve capability to understand and rationally plan these complicated interactions in future solid tumour chimeric antigen receptor -T cell treatment. 
T-cell innovations that can be combined and tweaked for the optimal efficacy and safety will continue to advance human health and provide new treatment options for diseases that are presently incurable.

\section{References}

1. Kowalski, J.; Rzyman, W.; Biernat, W.; Montesano, C.; Bernardini, R.; Marektrzonkowska, N. Adoptive Cell Therapy - Harnessing Antigen-Specific T cells to Target Solid Tumours. 2020, 1-30.

2. Zhylko, A.; Winiarska, M.; Graczyk-jarzynka, A. The Great War of Today : Modifications of CAR-T Cells to E ff ectively Combat Malignancies. 2020.

3. Rosenblatt, E.; Zubizarreta, E. RADIOTHERAPY IN CANCER CARE : FACING THE.

4. Lu, R.; Hwang, Y.; Liu, I.; Lee, C.; Tsai, H.; Li, H.; Wu, H. Development of therapeutic antibodies for the treatment of diseases. 2020, 1-30.

5. Roberto, R.B. Di; Castellanos-rueda, R.; Frey, S.; Egli, D.; Vazquez-lombardi, R.; Kapetanovic, E.; Kucharczyk, J.; Reddy, S.T. A Functional Screening Strategy for Engineering Chimeric Antigen Receptors with Reduced On-Target, Off-Tumor Activation. Mol. Ther. 2020, 28, 2564-2576, doi:10.1016/j.ymthe.2020.08.003.

6. Cells, C.A.R.T.; Benmebarek, M.; Karches, C.H.; Cadilha, B.L.; Lesch, S.; Endres, S.; Kobold, S. Killing Mechanisms of Chimeric Antigen Receptor. 2019, doi:10.3390/ijms20061283.

7. Jin, K.T.; Chen, B.; Liu, Y.Y.; Lan, H.R.; Yan, J.P. Monoclonal antibodies and chimeric antigen receptor ( CAR ) T cells in the treatment of colorectal cancer. Cancer Cell Int. 2021, 1-15, doi:10.1186/s12935-021-01763-9.

8. Quintarelli, C.; Orlando, D.; Boffa, I.; Guercio, M.; Assunta, V. Choice of costimulatory domains and of cytokines determines CAR T-cell activity in neuroblastoma.

Oncoimmunology 2018, 7, 1-16, doi:10.1080/2162402X.2018.1433518.

9. Zambrano-Zaragoza, M.L.; González-Reza, R.; Mendoza-Muñoz, N.; Miranda-Linares, 
V.; Bernal-Couoh, T.F.; Mendoza-Elvira, S.; Quintanar-Guerrero, D. Nanosystems in edible coatings: A novel strategy for food preservation. Int. J. Mol. Sci. 2018, 19, doi:10.3390/ijms19030705.

10. Ajina, A.; Maher, J. Europe PMC Funders Group Strategies to address chimeric antigen receptor tonic signalling; 2019; Vol. 17; ISBN 2071880919.

11. Pinel, S.; Thomas, N.; Boura, C.; Barberi-heyob, M.; Pinel, S.; Thomas, N.; Boura, C.; Approaches, M.B.; Pinel, S.; Thomas, N.; et al. Approaches to physical stimulation of metallic nanoparticles for glioblastoma treatment To cite this version : HAL Id : hal01935483 Approaches to physical stimulation of metallic nanoparticles for glioblastoma treatment. Adv. Drug Deliv. Rev. 2019, doi:10.1016/j.addr.2018.10.013.

12. Waldman, A.D.; Fritz, J.M.; Lenardo, M.J. A guide to cancer immunotherapy: from T cell basic science to clinical practice. Nat. Rev. Immunol. 2020, 20, doi:10.1038/s41577-0200306-5.

13. Jaspers, J.E.; Brentjens, R.J.; Sloan, M.; Cancer, K.; Sloan, M.; Cancer, K.; Program, C.; Sloan, M.; Cancer, K. HHS Public Access. 2017, 28, 83-91, doi:10.1016/j.pharmthera.2017.03.012.Development.

14. Zhang, Q.; Ping, J.; Huang, Z.; Zhang, X.; Zhou, J.; Wang, G.; Liu, S.; Ma, J. Review Article CAR-T Cell Therapy in Cancer : Tribulations and Road Ahead. 2020, 2020.

15. Caruso, H.G.; Heimberger, A.B.; Cooper, L.J.N. Steering CAR T cells to distinguish friend from foe. Oncoimmunology 2019, 0, 1-10, doi:10.1080/2162402X.2016.1271857.

16. Rafiq, S.; Hackett, C.S.; Brentjens, R.J. therapy. Nat. Rev. Clin. Oncol. 2020, 17, doi:10.1038/s41571-019-0297-y.

17. Marofi, F.; Motavalli, R.; Safonov, V.A.; Thangavelu, L.; Yumashev, A.V.; Alexander, M.; Shomali, N.; Chartrand, M.S.; Pathak, Y.; Jarahian, M. CAR T cells in solid tumors : challenges and opportunities. 2021, 1, 1-16.

18. Richards, D.M.; Sefrin, J.P.; Gieffers, C.; Hill, O.; Merz, C. Concepts for agonistic targeting of CD40 in immuno-oncology. Hum. Vaccines Immunother. 2020, 16, 377-387, doi:10.1080/21645515.2019.1653744. 
19. Stoiber, S.; Cadilha, B.L.; Benmebarek, M.-R.; Lesch, S.; Endres, S.; Kobold, S. Limitations in the Design of Chimeric Antigen Receptors for Cancer Therapy. Cells 2019, 8, 472, doi:10.3390/cells8050472.

20. Koristka, S.; Ziller-Walter, P.; Bergmann, R.; Arndt, C.; Feldmann, A.; Kegler, A.; Cartellieri, M.; Ehninger, A.; Ehninger, G.; Bornhäuser, M.; et al. Anti-CAR-engineered T cells for epitope-based elimination of autologous CAR T cells. Cancer Immunol. Immunother. 2019, 68, 1401-1415, doi:10.1007/s00262-019-02376-y.

21. Wen, H.; Qu, Z.; Yan, Y.; Pu, C.; Wang, C.; Jiang, H.; Hou, T.; Huo, Y. Preclinical safety evaluation of chimeric antigen receptor-modified T cells against CD19 in NSG mice. Ann. Transl. Med. 2019, 7, 735-735, doi:10.21037/atm.2019.12.03.

22. Andrea, A.E.; Chiron, A.; Bessoles, S.; Hacein-Bey-abina, S. Engineering next-generation car-t cells for better toxicity management. Int. J. Mol. Sci. 2020, 21, 1-25, doi:10.3390/ijms21228620.

23. Guedan, S.; Calderon, H.; Posey, A.D.; Maus, M. V. Engineering and Design of Chimeric Antigen Receptors. Mol. Ther. - Methods Clin. Dev. 2019, 12, 145-156, doi:10.1016/j.omtm.2018.12.009.

24. Strohl; Naso Bispecific T-Cell Redirection versus Chimeric Antigen Receptor (CAR)-T Cells as Approaches to Kill Cancer Cells. Antibodies 2019, 8, 41, doi:10.3390/antib8030041.

25. Han, X.; Wang, Y.; Wei, J.; Han, W. Multi-antigen-targeted chimeric antigen receptor T cells for cancer therapy. J. Hematol. Oncol. 2019, 12, 1-10, doi:10.1186/s13045-0190813-7.

26. Caliendo, F.; Dukhinova, M.; Siciliano, V. Engineered Cell-Based Therapeutics : Synthetic Biology Meets Immunology. 2019, 7, 1-8, doi:10.3389/fbioe.2019.00043.

27. Hughes-parry, H.E.; Cross, R.S.; Jenkins, M.R. The Evolving Protein Engineering in the Design of Chimeric Antigen Receptor T Cells. 2019, 1-15.

28. Bedard, P.L.; Hansen, A.R.; Ratain, M.J.; Siu, L.L.; Centre, M.C. HHS Public Access. 2017, 501, 355-364, doi:10.1038/nature12627.Tumour. 
29. Caswell, D.R.; Swanton, C. The role of tumour heterogeneity and clonal cooperativity in metastasis , immune evasion and clinical outcome. 2017, 1-9, doi:10.1186/s12916-0170900-y.

30. Beyes, S.; Bediaga, N.G.; Zippo, A. An Epigenetic Perspective on Intra-Tumour Heterogeneity : Novel Insights and New Challenges from Multiple Fields. 2021.

31. Tellez-gabriel, M.; Ory, B.; Lamoureux, F.; Heymann, M.; Heymann, D. Tumour Heterogeneity: The Key Advantages of Single-Cell Analysis. 2012, doi:10.3390/ijms17122142.

32. Zaman, R.; Islam, R.A.; Ibnat, N.; Othman, I.; Zaini, A.; Lee, C.Y.; Chowdhury, E.H. Current strategies in extending half-lives of therapeutic proteins. J. Control. Release 2019, 301, 176-189, doi:10.1016/j.jconrel.2019.02.016.

33. Cooperation, W.; Guo, F.; Cui, J. CAR-T in Cancer Treatment : Develop in SelfOptimization ,. 2021.

34. Mavilio, F. Designing Lentiviral Vectors for Gene Therapy of Genetic Diseases. 2021, 114.

35. Li, H.; Yang, Y.; Hong, W.; Huang, M.; Wu, M.; Zhao, X. Applications of genome editing technology in the targeted therapy of human diseases : mechanisms, advances and prospects. Signal Transduct. Target. Ther. 2020, doi:10.1038/s41392-019-0089-y.

36. Mo, F.; Duan, S.; Jiang, X.; Yang, X.; Hou, X.; Shi, W.; Carlos, C.J.J.; Liu, A.; Yin, S.; Wang, W.; et al. Nanobody-based chimeric antigen receptor T cells designed by CRISPR/Cas9 technology for solid tumor immunotherapy. Signal Transduct. Target. Ther. 2021, 6, doi:10.1038/s41392-021-00462-1.

37. Od, Z.; Condori, J.; Peterson, N.; Zhou, S.; Krenciute, G. Integration and Expression in T Cells : Protocol and Application for T-Cell Therapy. 2020.

38. Bair, S.M.; Porter, D.L. Accelerating chimeric antigen receptor therapy in chronic lymphocytic leukemia: The development and challenges of chimeric antigen receptor Tcell therapy for chronic lymphocytic leukemia. 2019, 10-17, doi:10.1002/ajh.25457. 
39. Redeker, A.; Arens, R. Improving adoptive T cell therapy: The particular role of T cell costimulation, cytokines, and post-transfer vaccination. Front. Immunol. 2016, 7, 1-17, doi:10.3389/fimmu.2016.00345.

40. Wiebking, V.; Lee, C.M.; Mostrel, N.; Lahiri, P.; Bak, R.; Bao, G.; Roncarolo, M.G.; Bertaina, A.; Porteus, M.H. Genome editing of donor-derived T cells to generate allogeneic chimeric antigen receptor-modified T cells: optimizing a $\beta$ T-cell-depleted haploidentical hematopoietic stem cell transplantation. Haematologica 2021, 106, 847858, doi:10.3324/haematol.2019.233882.

41. Razeghian, E.; Nasution, M.K.M.; Rahman, H.S.; Gardanova, Z.R. A deep insight into CRISPR / Cas9 application in CAR-T cell-based tumor immunotherapies. 2021, 7, 1-17.

42. Georgiadis, C.; Preece, R.; Nickolay, L.; Etuk, A.; Petrova, A.; Ladon, D.; Danyi, A.; Humphryes-kirilov, N.; Ajetunmobi, A.; Kim, D.; et al. Long Terminal Repeat CRISPRCAR-Coupled “ Universal” T Cells Mediate Potent Anti-leukemic Effects. Mol. Ther. 2018, 26, 1215-1227, doi:10.1016/j.ymthe.2018.02.025.

43. Barrueto, L.; Caminero, F.; Cash, L.; Makris, C.; Lamichhane, P.; Deshmukh, R.R. Resistance to Checkpoint Inhibition in Cancer Immunotherapy. Transl. Oncol. 2020, 13, 100738, doi:10.1016/j.tranon.2019.12.010.

44. Dummer, R.G. Anti-PD-1 and Anti-CTLA-4 Therapies in Cancer : Mechanisms of Action , efficacy , and Limitations. 2018, 8, 1-14, doi:10.3389/fonc.2018.00086.

45. Li, C.; Jiang, P.; Wei, S.; Xu, X.; Wang, J. Regulatory T cells in tumor microenvironment: new mechanisms, potential therapeutic strategies and future prospects. 2020, 1-23.

46. Qin, S.; Xu, L.; Yi, M.; Yu, S.; Wu, K. Novel immune checkpoint targets : moving beyond PD-1 and CTLA-4. 2019, 1-14.

47. $\quad$ Long, K.B.; Young, R.M.; Boesteanu, A.C.; Davis, M.M.; Melenhorst, J.J.; Lacey, S.F.; Degaramo, D.A.; Levine, B.L.; Fraietta, J.A. CAR T Cell Therapy of Non-hematopoietic Malignancies : Detours on the Road to Clinical Success. 2018, 9 , doi:10.3389/fimmu.2018.02740. 
48. Safarzadeh Kozani, P.; Safarzadeh Kozani, P.; Rahbarizadeh, F. CAR-T cell therapy in Tcell malignancies: Is success a low-hanging fruit? Stem Cell Res. Ther. 2021, 12, 1-17, doi:10.1186/s13287-021-02595-0.

49. Dexter, A.; Jr, P.; August, B. CAR T Cells in Solid Tumors : Blueprints for Building effective Therapies. 2018, 9, 1-20, doi:10.3389/fimmu.2018.01740.

50. Hou, A.J.; Chang, Z.L.; Lorenzini, M.H.; Zah, E.; Chen, Y.Y. TGF- b - responsive CART cells promote anti-tumor immune function. 2018, 75-86, doi:10.1002/btm2.10097.

51. Junttila, I.S. Tuning the Cytokine Responses : An Update on interleukin ( iL ) -4 and iL-13 Receptor Complexes. 2018, 9, doi:10.3389/fimmu.2018.00888.

52. Tavernier, S.J.; Athanasopoulos, V.; Verloo, P.; Behrens, G.; Staal, J.; Bogaert, D.J.; Naesens, L.; De Bruyne, M.; Van Gassen, S.; Parthoens, E.; et al. A human immune dysregulation syndrome characterized by severe hyperinflammation with a homozygous nonsense Roquin-1 mutation. Nat. Commun. 2019, 10, doi:10.1038/s41467-019-12704-6.

53. Yanagihara, Y.; Ikizawa, K.; Kajiwara, K. Functional significance of IL-4 receptor on B cells in IL-4-induced human IgE production. 1995, 2-8.

54. Gill, N.; Paltser, G.; Ashkar, A.A. Interleukin-15 expression affects homeostasis and function of B cells through NK cell-derived interferon- c. Cell. Immunol. 2009, 15, 1-6, doi:10.1016/j.cellimm.2009.03.010.

55. Isidoro-garcía, M.; Dávila, I.; Laffond, E.; Moreno, E.; Lorente, F.; González-sarmiento, R. polymorphisms in asthma : a case control study. 2005, 4, 1-7, doi:10.1186/1476-7961$3-15$.

56. Martinez, M.; Moon, E.K. CAR T Cells for Solid Tumors : New Strategies for Finding, Infiltrating, and Surviving in the Tumor Microenvironment. 2019, 10, 1-21, doi:10.3389/fimmu.2019.00128.

57. Rodriguez-garcia, A.; Palazon, A.; Noguera-ortega, E.; Jr, D.J.P.; Martin, F. CAR-T Cells Hit the Tumor Microenvironment : Strategies to Overcome Tumor Escape. 2020, 11, 117, doi:10.3389/fimmu.2020.01109. 
58. Ji, F.; Zhang, F.; Zhang, M.; Long, K.; Xia, M.; Lu, F.; Li, E.; Chen, J.; Li, J.; Chen, Z.; et al. Targeting the DNA damage response enhances CD70 CAR-T cell therapy for renal carcinoma by activating the cGAS-STING pathway. J. Hematol. Oncol. 2021, 14, 1-5, doi:10.1186/s13045-021-01168-1.

59. Morgan, M.A.; Schambach, A. Engineering CAR-T Cells for Improved Function Against Solid Tumors. Front. Immunol. 2018, 9, 2493, doi:10.3389/fimmu.2018.02493.

60. Tian, Y.; Li, Y.; Shao, Y.; Zhang, Y. Gene modification strategies for next- generation CAR T cells against solid cancers. 2020, 6, 1-16.

61. Poorebrahim, M.; Melief, J.; Coaña, Y.P. De; Wickström, S.L.; Cid-arregui, A.; Kiessling, R. Counteracting CAR T cell dysfunction. Oncogene 2021, 421-435, doi:10.1038/s41388020-01501-X.

62. Jin, L.; Cao, L.; Zhu, Y.; Cao, J.; Li, X.; Zhou, J.; Liu, B. Enhance anti-lung tumor efficacy of chimeric antigen receptor-T cells by ectopic expression of $\mathrm{C}-\mathrm{C}$ motif chemokine receptor 6. Sci. Bull. 2021, 66, 803-812, doi:10.1016/j.scib.2020.12.027.

63. Bell, M.; Gottschalk, S. Engineered Cytokine Signaling to Improve CAR T Cell Effector Function. 2021, 12, 1-16, doi:10.3389/fimmu.2021.684642.

64. Zhang, Z.; Miao, L.; Ren, Z.; Tang, F.; Li, Y.; Fantini, M.; Golubovskaya, V.; Biotechnologies, P. Gene-Edited Interleukin CAR-T Cells Therapy in the Treatment of Malignancies : Present and Future. 2021, 12, 1-13, doi:10.3389/fimmu.2021.718686.

65. Chmielewski, M. TRUCKS , the fourth-generation CAR T cells : Current developments and clinical translation. 2020, 1-9, doi:10.1002/acg2.84.

66. Ramachandran, S.; Verma, A.K.; Dev, K.; Goyal, Y.; Bhatt, D.; Alsahli, M.A.; Rahmani, A.H.; Almatroudi, A.; Almatroodi, S.A.; Alrumaihi, F.; et al. Review Article Role of Cytokines and Chemokines in NSCLC Immune Navigation and Proliferation. 2021, 2021.

67. Huemer, F.; Leisch, M.; Geisberger, R.; Melchardt, T.; Rinnerthaler, G.; Zaborsky, N.; Greil, R. Combination Strategies for Immune-Checkpoint Blockade and Response Prediction by Artificial Intelligence. 4, 1-35. 
68. Hassanpour, S.H.; Dehghani, M. Review of cancer from perspective of molecular. $J$. Cancer Res. Pract. 2017, 4, 127-129, doi:10.1016/j.jcrpr.2017.07.001.

69. Wu, P.; Gao, W.; Su, M.; Nice, E.C.; Zhang, W.; Lin, J. Adaptive Mechanisms of Tumor Therapy Resistance Driven by Tumor Microenvironment. 2021, 9, 1-23, doi:10.3389/fcell.2021.641469.

70. Gowrishankar, K.; Birtwistle, L.; Micklethwaite, K. Manipulating the tumor microenvironment by adoptive cell transfer of CAR T-cells. Mamm. Genome 2018, 0, 0, doi:10.1007/s00335-018-9756-5.

71. Bruno, A.; Salemme, V.; Centonze, G.; Cavallo, F.; De, P. The Crosstalk Between Tumor Cells and the Immune Microenvironment in Breast Cancer : Implications for Immunotherapy. 2021, 11, 1-20, doi:10.3389/fonc.2021.610303.

72. Liu, B.; Yan, L.; Zhou, M. Target selection of CAR T cell therapy in accordance with the TME for solid tumors. Am. J. Cancer Res. 2019, 9, 228-241.

73. Ware, M.B.; El-, B.F.; Lesinski, G.B. Mirage or long- - awaited oasis : cell responses in pancreatic cancer. 2020, doi:10.1136/jitc-2020-001100.

74. Dong, Y.; Wan, Z.; Gao, X.; Yang, G.; Liu, L. Reprogramming Immune Cells for Enhanced Cancer Immunotherapy: Targets and Strategies. Front. Immunol. 2021, 12, 114, doi:10.3389/fimmu.2021.609762.

75. Agliardi, G.; Liuzzi, A.R.; Hotblack, A.; De Feo, D.; Núñez, N.; Stowe, C.L.; Friebel, E.; Nannini, F.; Rindlisbacher, L.; Roberts, T.A.; et al. Intratumoral IL-12 delivery empowers CAR-T cell immunotherapy in a pre-clinical model of glioblastoma. Nat. Commun. 2021, 12, 1-11, doi:10.1038/s41467-020-20599-x.

76. Lanitis, E.; Kosti, P.; Ronet, C.; Cribioli, E.; Rota, G.; Spill, A.; Reichenbach, P.; Zoete, V.; Laniti, D.D.; Coukos, G.; et al. VEGFR-2 redirected CAR- - T cells are functionally impaired by soluble A competition for receptor binding. 2021, 1-13, doi:10.1136/jitc2020-002151.

77. Bedoya, D.M.; Dutoit, V.; Migliorini, D. Allogeneic CAR T Cells : An Alternative to Overcome Challenges of CAR T Cell Therapy in Glioblastoma. 2021, 12, 
doi:10.3389/fimmu.2021.640082.

78. Hematol, J.; Tian, Z.; Liu, M.; Zhang, Y.; Wang, X. Bispecific T cell engagers : an emerging therapy for management of hematologic malignancies. J. Hematol. Oncol. 2021, 1-18, doi:10.1186/s13045-021-01084-4.

79. Hematol, J.; Edeline, J.; Houot, R.; Marabelle, A.; Alcantara, M. CAR - T cells and BiTEs in solid tumors : challenges and perspectives. J. Hematol. Oncol. 2021, 1-12, doi:10.1186/s13045-021-01067-5. 